\title{
Azimuthal Charged-Particle Correlations and Possible Local Strong Parity Violation
}

B. I. Abelev, ${ }^{8}$ M. M. Aggarwal, ${ }^{29}$ Z. Ahammed ${ }^{46}$ A. V. Alakhverdyants, ${ }^{16}$ B. D. Anderson, ${ }^{17}$ D. Arkhipkin, ${ }^{3}$ G. S. Averichev, ${ }^{16}$ J. Balewski, ${ }^{21}$ O. Barannikova, ${ }^{8}$ L. S. Barnby, ${ }^{2}$ S. Baumgart, ${ }^{51}$ D. R. Beavis, ${ }^{3}$ R. Bellwied, ${ }^{49}$ F. Benedosso, ${ }^{26}$ M. J. Betancourt, ${ }^{21}$ R. R. Betts, ${ }^{8}$ A. Bhasin, ${ }^{15}$ A. K. Bhati, ${ }^{29}$ H. Bichsel,${ }^{48}$ J. Bielcik, ${ }^{10}$ J. Bielcikova, ${ }^{11}$ B. Biritz, ${ }^{6}$ L. C. Bland,${ }^{3}$ I. Bnzarov, ${ }^{16}$ B. E. Bonner,${ }^{35}$ J. Bouchet,${ }^{17}$ E. Braidot,${ }^{26}$ A. V. Brandin, ${ }^{24}$ A. Bridgeman, ${ }^{1}$ E. Bruna, ${ }^{51}$ S. Bueltmann, ${ }^{28}$ T. P. Burton, ${ }^{2}$ X. Z. Cai,${ }^{39}$ H. Caines, ${ }^{51}$ M. Calderón de la Barca Sánchez, ${ }^{5}$ O. Catu, ${ }^{51}$ D. Cebra,${ }^{5}$ R. Cendejas, ${ }^{6}$ M. C. Cervantes,${ }^{41}$ Z. Chajecki, ${ }^{27}$ P. Chaloupka, ${ }^{11}$ S. Chattopadhyay, ${ }^{46}$ H. F. Chen, ${ }^{37}$ J. H. Chen, ${ }^{17}$ J. Y. Chen, ${ }^{50}$ J. Cheng, ${ }^{43}$ M. Cherney, ${ }^{9}$ A. Chikanian, ${ }^{51}$ K. E. Choi,${ }^{33}$ W. Christie,${ }^{3}$ P. Chung, ${ }^{11}$ R. F. Clarke, ${ }^{41}$ M. J. M. Codrington, ${ }^{41}$ R. Corliss, ${ }^{21}$ T. M. Cormier, ${ }^{49}$ M. R. Cosentino, ${ }^{36}$ J. G. Cramer ${ }^{48}$ H. J. Crawford, ${ }^{4}$ D. Das, ${ }^{5}$ S. Dash, ${ }^{12}$ M. Daugherity, ${ }^{42}$ L. C. De Silva, ${ }^{49}$ T. G. Dedovich, ${ }^{16}$ M. DePhillips, ${ }^{3}$ A. A. Derevschikov, ${ }^{31}$ R. Derradi de Souza, ${ }^{7}$ L. Didenko, ${ }^{3}$ P. Djawotho, ${ }^{41}$ V. Dzhordzhadze ${ }^{3}$ S. M. Dogra, ${ }^{15}$ X. Dong, ${ }^{20}$ J. L. Drachenberg, ${ }^{41}$ J.E. Draper, ${ }^{5}$ J. C. Dunlop, ${ }^{3}$ M. R. Dutta Mazumdar, ${ }^{46}$ L. G. Efimov, ${ }^{16}$ E. Elhalhuli, ${ }^{2}$ M. Elnimr,${ }^{49}$ J. Engelage, ${ }^{4}$

G. Eppley, ${ }^{35}$ B. Erazmus,${ }^{40}$ M. Estienne, ${ }^{40}$ L. Eun, ${ }^{30}$ P. Fachini,${ }^{3}$ R. Fatemi, ${ }^{18}$ J. Fedorisin, ${ }^{16}$ A. Feng,${ }^{50}$ P. Filip, ${ }^{16}$ E. Finch, ${ }^{51}$ V. Fine,${ }^{3}$ Y. Fisyak, ${ }^{3}$ C. A. Gagliardi, ${ }^{41}$ D. R. Gangadharan, ${ }^{6}$ M. S. Ganti, ${ }^{46}$ E. J. Garcia-Solis,${ }^{8}$ A. Geromitsos,${ }^{40}$ F. Geurts, ${ }^{35}$ V. Ghazikhanian, ${ }^{6}$ P. Ghosh, ${ }^{46}$ Y. N. Gorbunov, ${ }^{9}$ A. Gordon, ${ }^{3}$ O. Grebenyuk, ${ }^{20}$ D. Grosnick, ${ }^{45}$ B. Grube, ${ }^{33}$ S. M. Guertin, ${ }^{6}$ K. S. F. F. Guimaraes,${ }^{36}$ A. Gupta, ${ }^{15}$ N. Gupta, ${ }^{15}$ W. Guryn,${ }^{3}$ B. Haag, ${ }^{5}$ T. J. Hallman, ${ }^{3}$ A. Hamed, ${ }^{41}$ J. W. Harris ${ }^{51}$ M. Heinz,${ }^{51}$ S. Heppelmann, ${ }^{30}$ A. Hirsch, ${ }^{32}$ E. Hjort, ${ }^{20}$ A. M. Hoffman, ${ }^{21}$ G. W. Hoffmann, ${ }^{42}$ D. J. Hofman, ${ }^{8}$ R. S. Hollis, ${ }^{8}$ H. Z. Huang, ${ }^{6}$ T. J. Humanic,${ }^{27}$ L. Huo,${ }^{41}$ G. Igo, ${ }^{6}$ A. Iordanova, ${ }^{8}$ P. Jacobs,${ }^{20}$ W. W. Jacobs,${ }^{14}$ P. Jakl, ${ }^{11}$ C. Jena, ${ }^{12}$ F. Jin, ${ }^{39}$ C. L. Jones, ${ }^{21}$ P. G. Jones, ${ }^{2}$ J. Joseph,${ }^{17}$ E. G. Judd, ${ }^{4}$ S. Kabana,${ }^{40}$ K. Kajimoto, ${ }^{42}$ K. Kang,${ }^{43}$ J. Kapitan,${ }^{11}$ K. Kauder, ${ }^{8}$ D. Keane, ${ }^{17}$ A. Kechechyan, ${ }^{16}$ D. Kettler, ${ }^{48}$ V. Yu. Khodyrev, ${ }^{31}$ D. P. Kikola, ${ }^{20}$ J. Kiryluk, ${ }^{20}$ A. Kisiel ${ }^{47}$ S. R. Klein ${ }^{20}$ A. G. Knospe, ${ }^{51}$ A. Kocoloski, ${ }^{21}$ D. D. Koetke, ${ }^{45}$ J. Konzer,${ }^{32}$ M. Kopytine, ${ }^{17}$ I. Koralt,${ }^{28}$ W. Korsch, ${ }^{18}$ L. Kotchenda, ${ }^{24}$ V. Kouchpil, ${ }^{11}$ P. Kravtsov, ${ }^{24}$ V. I. Kravtsov,${ }^{31}$ K. Krueger, ${ }^{1}$ M. Krus, ${ }^{10}$ L. Kumar, ${ }^{29}$ P. Kurnadi, ${ }^{6}$ M. A. C. Lamont, ${ }^{3}$ J. M. Landgraf, ${ }^{3}$ S. LaPointe, ${ }^{49}$ J. Lauret, ${ }^{3}$ A. Lebedev, ${ }^{3}$ R. Lednicky, ${ }^{16}$ C-H. Lee, ${ }^{33}$ J. H. Lee, ${ }^{3}$ W. Leight ${ }^{21}$ M. J. LeVine,${ }^{3}$ C. Li,${ }^{37}$ N. Li,${ }^{50}$ Y. Li ${ }^{43}$ G. Lin,${ }^{51}$ S. J. Lindenbaum,${ }^{25}$ M. A. Lisa, ${ }^{27}$ F. Liu,${ }^{50}$ H. Liu, ${ }^{5}$ J. Liu, ${ }^{35}$

L. Liu, ${ }^{50}$ T. Ljubicic, ${ }^{3}$ W. J. Llope,${ }^{35}$ R. S. Longacre, ${ }^{3}$ W. A. Love,${ }^{3}$ Y. Lu ${ }^{37}$ T. Ludlam, ${ }^{3}$ G. L. Ma, ${ }^{39}$ Y. G. Ma, ${ }^{39}$ D. P. Mahapatra, ${ }^{12}$ R. Majka,${ }^{51}$ O. I. Mall,,${ }^{5}$ L. K. Mangotra, ${ }^{15}$ R. Manweiler, ${ }^{45}$ S. Margetis,${ }^{17}$ C. Markert ${ }^{42}$ H. Masui, ${ }^{20}$ H. S. Matis, ${ }^{20}$ Yu. A. Matulenko, ${ }^{31}$ D. McDonald,${ }^{35}$ T. S. McShane, ${ }^{9}$ A. Meschanin, ${ }^{31}$ R. Milner, ${ }^{21}$ N. G. Minaev, ${ }^{31}$ S. Mioduszewski, ${ }^{41}$ A. Mischke, ${ }^{26}$ B. Mohanty, ${ }^{46}$ D. A. Morozov, ${ }^{31}$ M. G. Munhoz,${ }^{36}$ B. K. Nandi, ${ }^{13}$ C. Nattrass, ${ }^{51}$ T. K. Nayak, ${ }^{46}$ J. M. Nelson, ${ }^{2}$ P. K. Netrakanti, ${ }^{32}$ M. J. Ng, ${ }^{4}$ L. V. Nogach, ${ }^{31}$ S. B. Nurushev, ${ }^{31}$ G. Odyniec,${ }^{20}$ A. Ogawa, ${ }^{3}$ H. Okada, ${ }^{3}$ V. Okorokov, ${ }^{24}$ D. Olson,${ }^{20}$ M. Pachr, ${ }^{10}$ B. S. Page,${ }^{14}$ S. K. Pal,${ }^{46}$ Y. Pandit, ${ }^{17}$ Y. Panebratsev, ${ }^{16}$ T. Pawlak, ${ }^{47}$ T. Peitzmann, ${ }^{26}$ V. Perevoztchikov, ${ }^{3}$ C. Perkins, ${ }^{4}$ W. Peryt, ${ }^{47}$ S. C. Phatak, ${ }^{12}$ P. Pile, ${ }^{3}$ M. Planinic,${ }^{52}$ M. A. Ploskon, ${ }^{20}$ J. Pluta, ${ }^{47}$ D. Plyku, ${ }^{28}$ N. Poljak, ${ }^{52}$ A. M. Poskanzer, ${ }^{20}$ B. V. K. S. Potukuchi, ${ }^{15}$ D. Prindle, ${ }^{48}$ C. Pruneau,${ }^{49}$ N. K. Pruthi, ${ }^{29}$

P. R. Pujahari, ${ }^{13}$ J. Putschke, ${ }^{51}$ R. Raniwala ${ }^{34}$ S. Raniwala, ${ }^{34}$ R. L. Ray,${ }^{42}$ R. Redwine, ${ }^{21}$ R. Reed,${ }^{5}$ A. Ridiger, ${ }^{24}$ H. G. Ritter, ${ }^{20}$ J. B. Roberts, ${ }^{35}$ O. V. Rogachevskiy, ${ }^{16}$ J. L. Romero, ${ }^{5}$ A. Rose, ${ }^{20}$ C. Roy, ${ }^{40}$ L. Ruan, ${ }^{3}$ M. J. Russcher ${ }^{26}$ R. Sahoo, ${ }^{40}$ S. Sakai, ${ }^{6}$ I. Sakrejda ${ }^{20}$ T. Sakuma, ${ }^{21}$ S. Salur, ${ }^{20}$ J. Sandweiss, ${ }^{51}$ J. Schambach,${ }^{42}$ R. P. Scharenberg, ${ }^{32}$ N. Schmitz, ${ }^{22}$ J. Seele, ${ }^{21}$ J. Seger, ${ }^{9}$ I. Selyuzhenkov, ${ }^{14}$ Y. Semertzidis, ${ }^{3}$ P. Seyboth, ${ }^{22}$ E. Shahaliev, ${ }^{16}$ M. Shao, ${ }^{37}$ M. Sharma ${ }^{49}$ S. S. Shi ${ }^{50}$ X-H. Shi, ${ }^{39}$ E. P. Sichtermann, ${ }^{20}$ F. Simon, ${ }^{22}$ R. N. Singaraju ${ }^{46}$ M. J. Skoby, ${ }^{32}$ N. Smirnov,${ }^{51}$ P. Sorensen, ${ }^{3}$ J. Sowinski, ${ }^{14}$ H. M. Spinka, ${ }^{1}$ B. Srivastava, ${ }^{32}$ T. D. S. Stanislaus,${ }^{45}$ D. Staszak,${ }^{6}$ M. Strikhanov, ${ }^{24}$ B. Stringfellow, ${ }^{32}$ A. A. P. Suaide, ${ }^{36}$ M. C. Suarez,${ }^{8}$ N. L. Subba, ${ }^{17}$ M. Sumbera, ${ }^{11}$ X. M. Sun,${ }^{20}$ Y. Sun, ${ }^{37}$ Z. Sun, ${ }^{19}$

B. Surrow, ${ }^{21}$ T. J. M. Symons, ${ }^{20}$ A. Szanto de Toledo, ${ }^{36}$ J. Takahashi, ${ }^{7}$ A. H. Tang, ${ }^{3}$ Z. Tang,${ }^{37}$ L. H. Tarini, ${ }^{49}$

T. Tarnowsky, ${ }^{23}$ D. Thein,${ }^{42}$ J. H. Thomas, ${ }^{20}$ J. Tian, ${ }^{39}$ A. R. Timmins,${ }^{49}$ S. Timoshenko, ${ }^{24}$ D. Tlusty, ${ }^{11}$ M. Tokarev, ${ }^{16}$ V. N. Tram ${ }^{20}$ S. Trentalange, ${ }^{6}$ R. E. Tribble, ${ }^{41}$ O. D. Tsai, ${ }^{6}$ J. Ulery, ${ }^{32}$ T. Ullrich, ${ }^{3}$ D. G. Underwood, ${ }^{1}$ G. Van Buren, ${ }^{3}$ G. van Nieuwenhuizen, ${ }^{21}$ J. A. Vanfossen, Jr., ${ }^{17}$ R. Varma, ${ }^{13}$ G. M. S. Vasconcelos, ${ }^{7}$ A. N. Vasiliev, ${ }^{31}$ F. Videbaek, ${ }^{3}$ Y.P. Viyogi, ${ }^{46}$ S. Vokal, ${ }^{16}$ S. A. Voloshin, ${ }^{49}$ M. Wada ${ }^{42}$ M. Walker, ${ }^{21}$ F. Wang,${ }^{32}$ G. Wang, ${ }^{6}$ H. Wang, ${ }^{23}$ J. S. Wang, ${ }^{19}$

Q. Wang, ${ }^{32}$ X. Wang, ${ }^{43}$ X.L. Wang, ${ }^{37}$ Y. Wang, ${ }^{43}$ G. Webb, ${ }^{18}$ J.C. Webb, ${ }^{45}$ G. D. Westfall, ${ }^{23}$ C. Whitten, Jr., ${ }^{6}$ H. Wieman, ${ }^{20}$ S. W. Wissink, ${ }^{14}$ R. Witt, ${ }^{44}$ Y. Wu, ${ }^{50}$ W. Xie, ${ }^{32}$ N. Xu, ${ }^{20}$ Q. H. Xu, ${ }^{38}$ Y. Xu, ${ }^{37}$ Z. Xu, ${ }^{3}$ Y. Yang, ${ }^{19}$ P. Yepes,${ }^{35}$ K. Yip, ${ }^{3}$ I-K. Yoo, ${ }^{33}$ Q. Yue ${ }^{43}$ M. Zawisza, ${ }^{47}$ H. Zbroszczyk, ${ }^{47}$ W. Zhan, ${ }^{19}$ S. Zhang, ${ }^{39}$ W. M. Zhang, ${ }^{17}$ X. P. Zhang, ${ }^{20}$ Y. Zhang, ${ }^{20}$ Z. P. Zhang, ${ }^{37}$ Y. Zhao, ${ }^{37}$ C. Zhong, ${ }^{39}$ J. Zhou, ${ }^{35}$ X. Zhu, ${ }^{43}$ R. Zoulkarneev, ${ }^{16}$

$$
\text { Y. Zoulkarneeva, }{ }^{16} \text { and J. X. Zuo }{ }^{39}
$$


(STAR Collaboration)

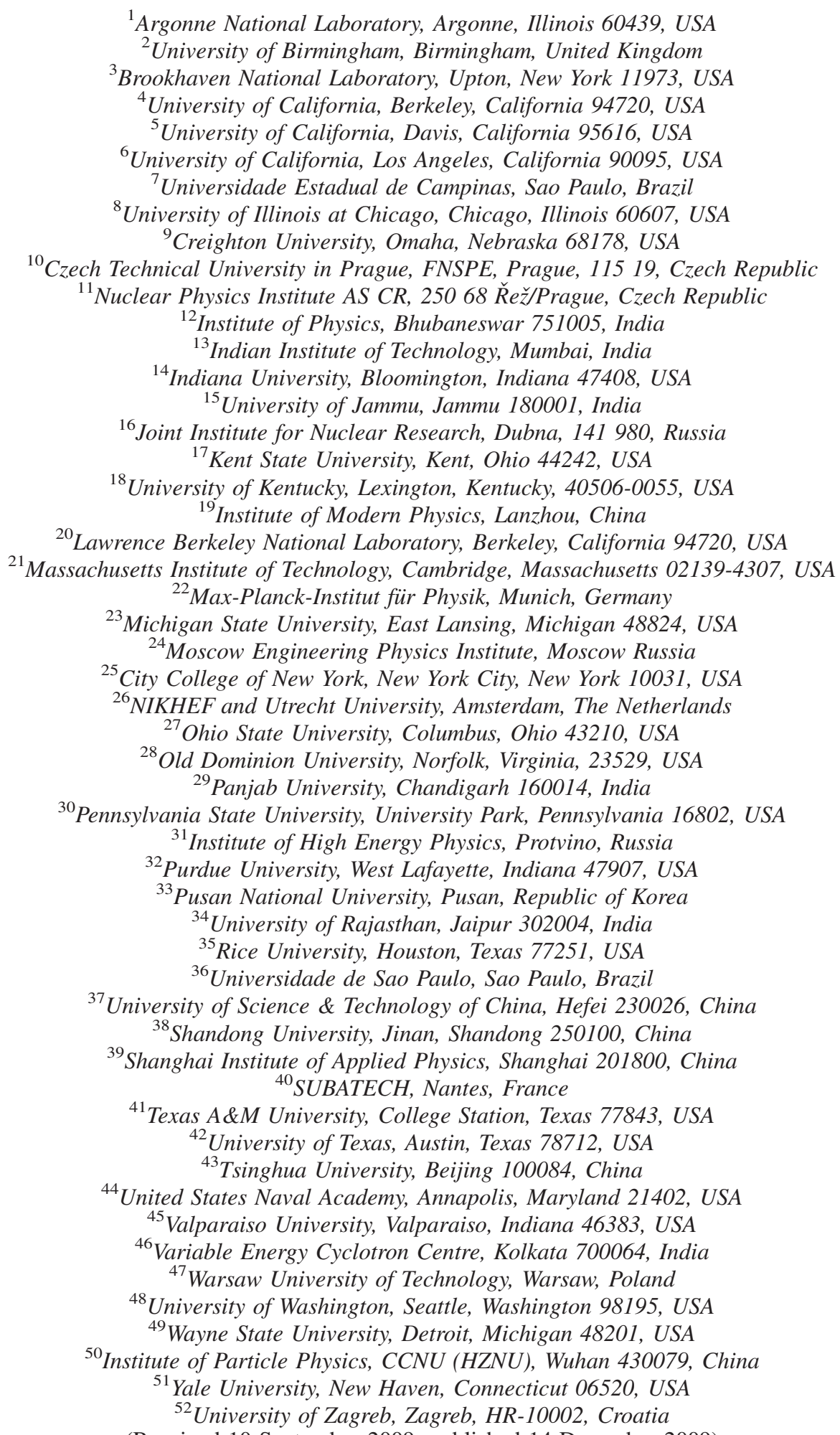

(Received 10 September 2009; published 14 December 2009)

Parity-odd domains, corresponding to nontrivial topological solutions of the QCD vacuum, might be created during relativistic heavy-ion collisions. These domains are predicted to lead to charge separation of quarks along the system's orbital momentum axis. We investigate a three-particle azimuthal correlator 
which is a $\mathcal{P}$ even observable, but directly sensitive to the charge separation effect. We report measurements of charged hadrons near center-of-mass rapidity with this observable in $\mathrm{Au}+\mathrm{Au}$ and $\mathrm{Cu}+$ $\mathrm{Cu}$ collisions at $\sqrt{s_{N N}}=200 \mathrm{GeV}$ using the STAR detector. A signal consistent with several expectations from the theory is detected. We discuss possible contributions from other effects that are not related to parity violation.

DOI: 10.1103/PhysRevLett.103.251601

Parity $(\mathcal{P})$ violation in the weak interaction was observed in 1957 [1]. However, until recently, parity has been thought to be conserved in the strong interaction. Modern QCD theory does allow for parity violation, but experiments have not seen this violation and the resulting constraints are tight $[2,3]$. Recently, it has been suggested that the hot and dense matter created in heavy-ion collisions may form metastable domains where the parity and time-reversal symmetries are locally violated [4]. In noncentral collisions, these domains may manifest themselves by giving positively and negatively charged particles opposite-direction momentum 'kicks' along the angular momentum vector of the collision. The resulting charge separation is a consequence of two factors [5-7]: the difference in numbers of quarks with positive and negative chiralities due to a nonzero topological charge of the metastable region, and the interaction of these particles with the extremely strong magnetic field produced in such a collision (leading to the effect being called the "Chiral Magnetic Effect"). This separation of charges along the angular momentum vector would be a clear $\mathcal{P}$ violation.

The expectation from this local $\mathcal{P}$ violation is that the relative sign of charge separation and angular momentum vectors is random in each event. This implies that any $\mathcal{P}$-odd observable should yield zero when averaged over many events. An experimental search for this effect must therefore involve comparing the measured charge separation signal in each event with the expected fluctuations due to non- $\mathcal{P}$-violating effects, or equivalently measuring correlations among particles in each event. This Letter reports the result of such a search performed in $200 \mathrm{GeV} \mathrm{Au}+\mathrm{Au}$ and $\mathrm{Cu}+\mathrm{Cu}$ heavy-ion collisions with the STAR detector at the Relativistic Heavy Ion Collider (RHIC).

Observables and method.-In an event, charge separation along the angular momentum vector may be described phenomenologically by sine terms in the Fourier decomposition of the charged-particle azimuthal distribution

$$
\begin{gathered}
\frac{d N_{ \pm}}{d \phi} \propto 1+2 v_{1} \cos \left(\phi-\Psi_{\mathrm{RP}}\right)+2 v_{2} \cos \left(2\left(\phi-\Psi_{\mathrm{RP}}\right)\right) \\
+\ldots+2 a_{ \pm} \sin \left(\phi-\Psi_{\mathrm{RP}}\right)+\ldots
\end{gathered}
$$

where $\Psi_{\mathrm{RP}}$ is the azimuthal angle of the reaction plane (the plane which contains the impact parameter and beam momenta-see Fig. 1 for a schematic). $v_{1}$ and $v_{2}$ are coefficients accounting for the so-called directed and elliptic flow [8]. The $a$ parameters, $a_{-}=-a_{+}$, describe the $\mathcal{P}$-violating effect. The spontaneous nature of the predicted
PACS numbers: 11.30.Er, 11.30.Qc, 12.38.Aw, 25.75.Nq

parity violation means that the sign of $a_{+}$and $a_{-}$vary from event to event and on average $\left\langle a_{+}\right\rangle=\left\langle a_{-}\right\rangle=0$.

We may, however, expect nonzero values for the correlator $\left\langle a_{\alpha} a_{\beta}\right\rangle$ (where $\alpha, \beta$ represent electric charge + or - ) since $\mathcal{P}$-violating contributions to these observables would accumulate over many events. One could measure $\left\langle a_{\alpha} a_{\beta}\right\rangle$ by calculating the average $\left\langle\sin \Delta \phi_{\alpha} \sin \Delta \phi_{\beta}\right\rangle$ over all particles of charge $\alpha$ paired with all particles of charge $\beta$ (here we have introduced the notation $\Delta \phi=\phi-\Psi_{R P}$ ). This is, however, also sensitive to several parity conserving physics backgrounds which produce correlations that have nonzero projections along the angular momentum vector. This led to the proposal [9] of the observable $\left\langle\cos \left(\phi_{\alpha}+\phi_{\beta}-2 \Psi_{\mathrm{RP}}\right)\right\rangle=\left(\left\langle\cos \Delta \phi_{\alpha} \cos \Delta \phi_{\beta}\right\rangle-\right.$ $\left.\left\langle\sin \Delta \phi_{\alpha} \sin \Delta \phi_{\beta}\right\rangle\right)$ which is sensitive to $-\left\langle a_{\alpha} a_{\beta}\right\rangle$. This observable represents the difference between azimuthal correlations projected onto the direction of the angular momentum vector and correlations projected onto the collision reaction plane. By taking this difference, these parity conserving correlations only produce backgrounds inasmuch as they depend on orientation with respect to the reaction plane. As a consequence of the $\left\langle\cos \Delta \phi_{\alpha} \cos \Delta \phi_{\beta}\right\rangle$ term, this observable is also sensitive to contributions from directed flow and its fluctuations. Because the measure-

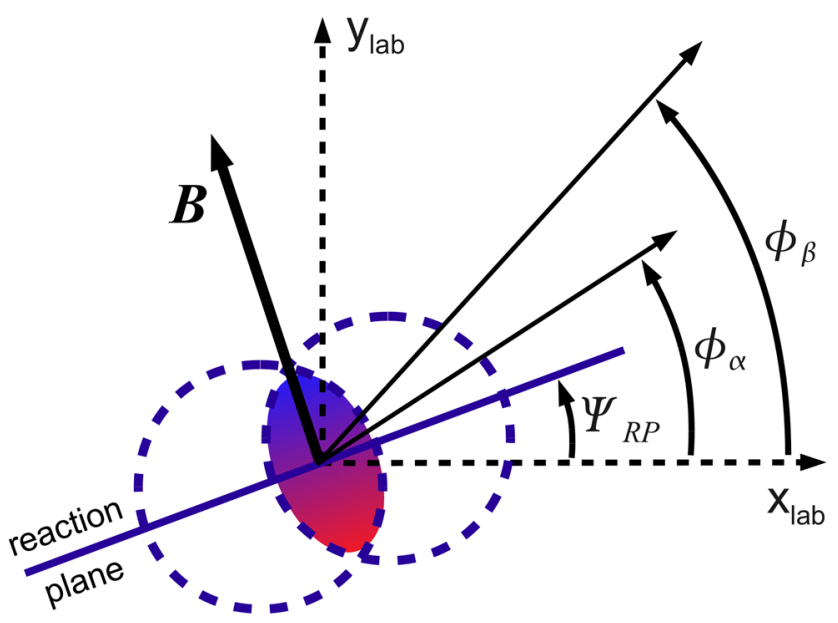

FIG. 1 (color online). Schematic depiction of the transverse plane in a collision of two heavy ions (shown as dotted outlines-one emerging from and one going into the page). The azimuthal angles of the reaction plane and produced particles with charges $\alpha$ and $\beta$ as used in Eqs. (1) and (2) are depicted here. 
ments presented in this Letter are for a symmetric rapidity region around center-of-mass rapidity, these contributions are negligible [10].

In practice, the reaction plane of a heavy-ion collision is not known, and one estimates it by measuring the "event plane" which is reconstructed from particle azimuthal distributions [8]. In the present analysis, this is done by using three-particle azimuthal correlations in which the third particle (labeled " $c$ " below) serves to measure the event plane $[9,11]$. Assuming that the only correlation between particles of type " $c$ " and particles $\alpha, \beta$ is through the common correlation to the reaction plane, we can evaluate our observable for $-\left\langle a_{\alpha} a_{\beta}\right\rangle$ as

$$
\left\langle\cos \left(\phi_{a}+\phi_{\beta}-2 \Psi_{\mathrm{RP}}\right)\right\rangle=\left\langle\cos \left(\phi_{a}+\phi_{\beta}-2 \phi_{c}\right)\right\rangle / v_{2, c} .
$$

By varying our choice of the type and momentum range of the " $c$ " particles, we have tested this assumption as described below.

Based on available theoretical understanding of the chiral magnetic effect we expect the following features of the correlator: neglecting any "final state" interaction with the medium, one expects $\left\langle a_{+} a_{+}\right\rangle=\left\langle a_{-} a_{-}\right\rangle=-\left\langle a_{+} a_{-}\right\rangle>0$. The likely effect of final state interactions in a hot dense medium [6] is a suppression of back-to-back correlations (i.e., correlations among two particles that travel in opposite directions through the medium), causing $\left\langle a_{+} a_{+}\right\rangle \gg$ $\left|\left\langle a_{+} a_{-}\right\rangle\right|$. The dependence of the signal on the size of the colliding system has not yet been calculated, but one qualitative prediction is that the suppression of oppositecharge correlations should be smaller in collisions of lighter nuclei [6]. For a given collision system, under the assumption that the average size of the $\mathcal{P}$-violating domain does not change with centrality [6], the correlator should be inversely proportional to charged-particle multiplicity, $N_{c h}$, scaled by a factor accounting for the magnetic field in the collisions. Finally, because the phenomenon is nonperturbative in nature, we expect that the signal should not extend in transverse momentum far beyond $1 \mathrm{GeV} / c$, although this may be affected by radial flow of the produced particles.

Data and detector--The data were obtained with the STAR detector [12] during RHIC runs in 2003-04 and 2004-05. The results are based on 14.7 M Au + Au and 13.9 M Cu$+\mathrm{Cu}$ events at $\sqrt{s_{\mathrm{NN}}}=200 \mathrm{GeV}$. A minimum bias trigger was used with events sorted into centrality classes based upon charged-particle multiplicity.

The correlations are reported for charged-particle tracks measured in the STAR time projection chamber (TPC) with pseudorapidity $|\eta|<1.0$ and transverse momentum $0.15<p_{t}<2 \mathrm{GeV} / c$. For event plane determination, in addition to the main TPC we use measurements in the two Forward TPCs $(2.7<|\eta|<3.9)$ and two zero degree calorimeter shower maximum detectors (ZDC-SMDs) [13].
The latter are sensitive to the directed flow of neutrons in the beam rapidity region.

The STAR detector is well suited to measure azimuthal correlations. The TPC has full azimuthal coverage and a charged-particle track reconstruction efficiency of approximately $85 \%$. Nevertheless, TPC sector boundaries, occasional readout channel outages, etc., may introduce biases in the analysis. In particular, they may cause inefficiencies that are different for positive and negative particles. In evaluating Eq. (2), we correct for detector effects (following $[14,15])$ by replacing $\cos \left(n \phi_{i}\right)$ with $\left[\cos \left(n \phi_{i}\right)-\right.$ $\langle\cos (n \phi)\rangle]$ for each particle, and similarly for the $\sin \left(n \phi_{i}\right)$ terms which also appear [11]. We calculate the "recentering" corrections $\langle\cos (n \phi)\rangle$ and $\langle\sin (n \phi)\rangle$ as a function of time as well as event multiplicity and $z$-vertex position. We also account for the acceptance dependence on particle $\eta, p_{t}$ and charge. Higher order acceptance corrections are found to be negligible.

Experimental uncertainties. - The dominant experimental systematic error comes from our knowledge of $v_{2}$ which is used in Eq. (2). The shaded bands in the figures reflect this systematic error with the actual points determined by applying $v_{2}$ for TPC particles as measured using the reaction plane found in the FTPC.

Other experimental systematics, including possible biases due to acceptance and detector efficiency and errors related to track quality cuts, are found to be comparable to or smaller than statistical errors [11].

Results.-Figure 2 presents $\left\langle\cos \left(\phi_{\alpha}+\phi_{\beta}-2 \Psi_{\mathrm{RP}}\right)\right\rangle$ for $\mathrm{Au}+\mathrm{Au}$ and $\mathrm{Cu}+\mathrm{Cu}$ collisions at $\sqrt{s_{\mathrm{NN}}}=$ $200 \mathrm{GeV}$ as evaluated using the right-hand side of Eq. (2) (error bars indicate statistical errors). The signal in $\mathrm{Cu}+\mathrm{Cu}$ collisions is larger than the signal in $\mathrm{Au}+\mathrm{Au}$ collisions at the same centrality, qualitatively consistent with the expected decrease of the signal with increasing multiplicity. For the $\mathrm{Au}+\mathrm{Au}$ system, opposite-charge correlations are clearly smaller in magnitude than samecharge correlations, in qualitative agreement with the possible suppression of back-to-back charge correlations. This is supported by the observation of a smaller difference in magnitude between same-charge and opposite-charge correlations in the smaller $\mathrm{Cu}+\mathrm{Cu}$ system. However, there is a large potential background contribution from threeparticle clusters to opposite-charge correlations which is discussed below and indicated by the thick solid $(\mathrm{Au}+\mathrm{Au})$ and dashed $(\mathrm{Cu}+\mathrm{Cu})$ lines on Fig. 2.

Figure 3 shows the dependence of the signal on the sum of the transverse momentum magnitudes of the two particles for the $30 \%-50 \%$ centrality range in $200 \mathrm{GeV} \mathrm{Au}+$ $\mathrm{Au}$ collisions. We do not observe a saturation or drop of the magnitude of the signal at high $p_{t}$ as one might naively expect for local $\mathcal{P}$ violation. The correlations are nearly independent of the $p_{t}$ difference over the range $0<\mid p_{t, \alpha}-$ $p_{t, \beta} \mid<2 \mathrm{GeV} / c$ [11], which excludes quantum interference (HBT) or Coulomb effects as possible explanations 


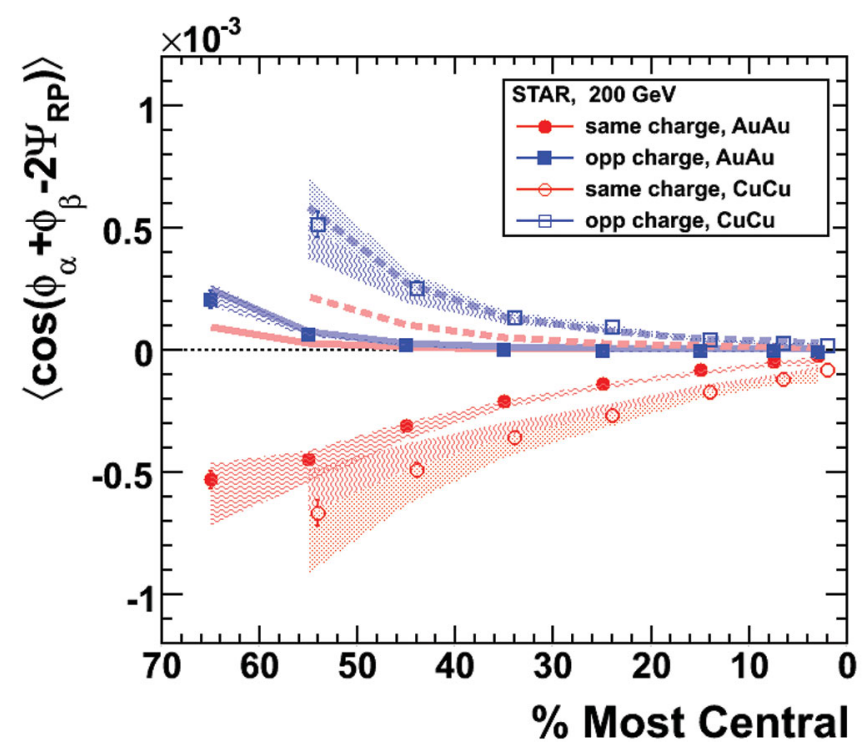

FIG. 2 (color). $\left\langle\cos \left(\phi_{a}+\phi_{\beta}-2 \Psi_{\mathrm{RP}}\right)\right\rangle$ in $\mathrm{Au}+\mathrm{Au}$ and $\mathrm{Cu}+\mathrm{Cu}$ collisions at $\sqrt{s_{\mathrm{NN}}}=200 \mathrm{GeV}$ calculated using Eq. (2). The thick solid $(\mathrm{Au}+\mathrm{Au})$ and dashed $(\mathrm{Cu}+\mathrm{Cu})$ lines represent HIJING calculations of the contributions from threeparticle correlations. Shaded bands represent uncertainty from the measurement of $v_{2}$. Collision centrality increases from left to right.

for the signal. We have studied the dependence of the signal on $\left|\eta_{\alpha}-\eta_{\beta}\right|$ [11], and find that the signal has a width of about one unit of $\eta$.

Physics backgrounds.-We first consider backgrounds due to multiparticle correlations (3 or more particles) which are not related to the reaction plane. This contribu-

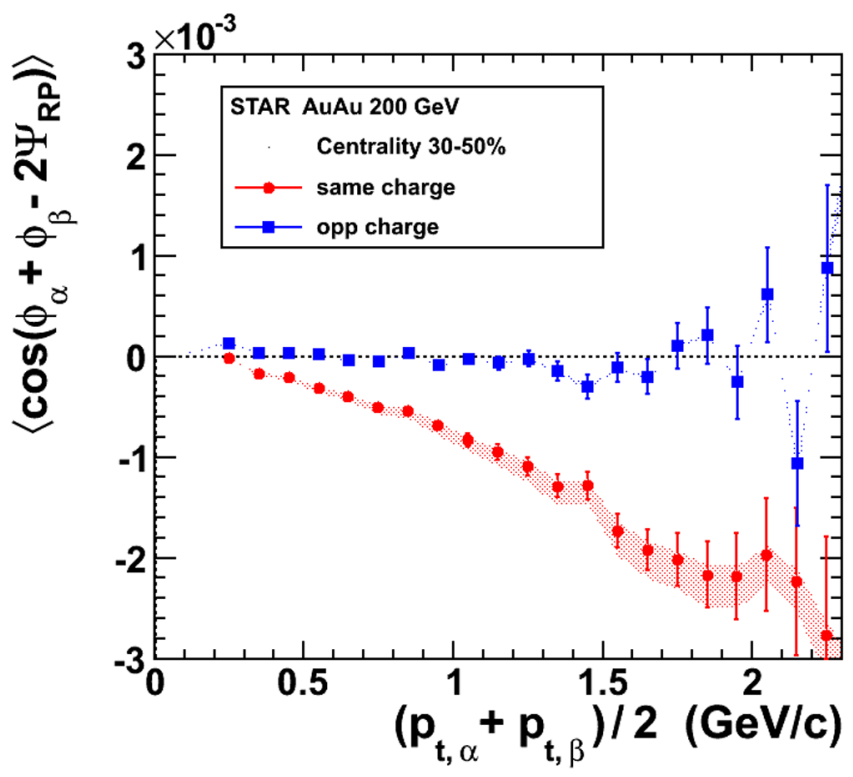

FIG. 3 (color online). Dependence of $\left\langle\cos \left(\phi_{\alpha}+\phi_{\beta}-\right.\right.$ $\left.\left.2 \Psi_{R P}\right)\right\rangle$ on $\frac{1}{2}\left(p_{t, \alpha}+p_{t, \beta}\right)$ calculated using no upper cut on particles' $p_{t}$. Shaded bands represent $v_{2}$ uncertainty. tion affects the assumption that two particle correlations with respect to the reaction plane [left-hand side of Eq. (2)] can be evaluated in practice via three-particle correlations [right-hand side of Eq. (2)]. Evidence supporting this assumption comes from the consistency of same-charge results when the reaction plane is found using particles " $c$ " detected in the TPC, FTPC, or ZDC-SMD, though the FTPC and (particularly) ZDC-SMD analyses have large statistical errors in the most peripheral bins. This multiparticle background should be negligible when the ZDCSMD event plane is used, so it can certainly be reduced and this is an important goal of future high statistics runs. To study these backgrounds in the current analysis, we use the heavy-ion event model HIJING [16] (used with default settings and jet quenching off in all calculations shown in this Letter) which includes production and fragmentation of mini jets. We find that the contribution to oppositecharge correlations of three-particle correlations in HIJING (represented by the thick solid and dashed lines in Figs. 2 and 4) is similar to the measured signal in several peripheral bins. We thus cannot conclude that there is an opposite-charge signal above possible background. The same-charge signal predicted by three-particle correlations in HIJING is much smaller and of opposite sign compared to that seen in the data.

Another class of backgrounds (which cannot be reduced by better determination of the reaction plane) consists of

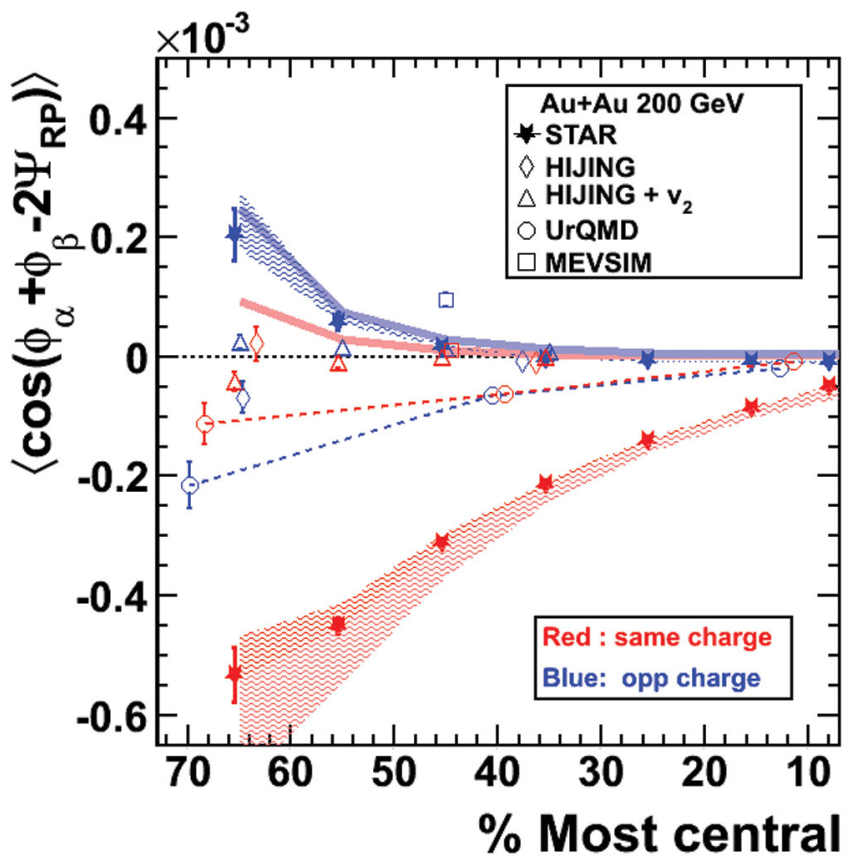

FIG. 4 (color). $\left\langle\cos \left(\phi_{\alpha}+\phi_{\beta}-2 \Psi_{\mathrm{RP}}\right)\right\rangle$ results from $200 \mathrm{GeV}$ $\mathrm{Au}+\mathrm{Au}$ collisions are compared to calculations with event generators HIJING (with and without an "elliptic flow afterburner"), URQMD (connected by dashed lines), and MEVSIM. Thick lines represent HIJING reaction-plane-independent background. 
processes in which particles $\alpha$ and $\beta$ are products of a cluster of two or more particles (for example a resonance decay or jet) and the cluster itself exhibits elliptic flow or fragments differently when emitted in-plane compared to out-of-plane [9,11].

For jets with a leading charged particle of $p_{t}>$ $3 \mathrm{GeV} / c$, we estimate the contribution using previous STAR measurements $[17,18]$ and find it to be negligible. To extend the study to lower $p_{t}$, we rely on HIJING calculations of two particle correlations with respect to the true reaction plane. These calculations also predict the contribution to be small compared to our measured signal as shown by the triangles in Fig. 4.

Resonance decays have the potential to contribute to $\left\langle\cos \left(\phi_{\alpha}+\phi_{\beta}-2 \Psi_{\mathrm{RP}}\right)\right\rangle$. In addition, previous correlation measurements from the ISR [19] and RHIC [20,21] indicate that a prominent role in particle production is played by clusters. A much smaller signal is expected for samethan opposite-charge correlations from resonances, which is qualitatively very unlike the signal shown in Fig. 2. Kinematic studies demonstrate that it is very difficult for the correct sign of fake signal to be created in the samecharge correlations without postulating a negative value of $v_{2}$ for the resonances or particles from cluster decays.

To search for other backgrounds to $\left\langle\cos \left(\phi_{\alpha}+\phi_{\beta}-\right.\right.$ $\left.\left.2 \Psi_{\mathrm{RP}}\right)\right\rangle$, we have simulated $\mathrm{Au}+\mathrm{Au}$ collisions with heavy-ion event generators MEVSIM [22], URQMD [23], and HIJING (with and without an elliptic flow afterburner implemented as suggested in [8]) for comparison and these results (calculated using the true reaction plane in all cases) are shown as open symbols in Fig. 4. MEVSIM only includes correlations due to resonance decays and an overall elliptic flow pattern. URQMD and HIJING are real physics models of the collision and so include correlations from many different physical processes. Figure 4 shows that no generator gives qualitative agreement with data for two particle correlations with respect to the reaction plane. The models also do not match the measured values for reaction-planeindependent correlations, $\left\langle\cos \left(\phi_{\alpha}-\phi_{\beta}\right)\right\rangle$ [11].

Other effects which produce insignificant contributions [11] include global polarization of hyperons along the direction of the system angular momentum.

Summary.-Measurements of three-particle correlations that are directly sensitive to predicted local $\mathcal{P}$-violation in heavy-ion collisions have been presented for $\mathrm{Au}+\mathrm{Au}$ and $\mathrm{Cu}+\mathrm{Cu}$ collisions at $\sqrt{s_{N N}}=200 \mathrm{GeV}$ as a function of collision centrality and (in $\mathrm{Au}+\mathrm{Au}$ ) particle transverse momentum, with a more complete set of measurements reported in [11].

Qualitatively the results agree with the magnitude and gross features of the theoretical predictions for local $\mathcal{P}$-violation in heavy-ion collisions, but the signal persists to higher transverse momentum than expected. The observable used in our analysis is $\mathcal{P}$ even and might be sensitive to non-parity-violating effects. So far, with the systematics checks discussed in this paper and [11], we have not identified effects that would explain the observed same-charge correlations. The observed signal cannot be described by the background models that we have studied (HIJING, HIJING $+v_{2}$, URQMD, MEVSIM), which span a broad range of hadronic physics.

A number of future experiments and analyses are naturally suggested by these results. One of them is the dependence of the signal on the collision energy. The charge separation effect is expected to depend strongly on the formation of a quark-gluon plasma [6], and the signal might be greatly suppressed or completely absent at an energy below that at which a QGP can be formed.

Improved theoretical calculations of the expected signal and potential physics backgrounds in high energy heavyion collisions are essential to understand whether or not the observed signal is due to local strong parity violation, and to further experimental study of this phenomenon.

We thank D. Kharzeev for many helpful discussions. We thank the RHIC Operations Group and RCF at BNL, the NERSC Center at LBNL and the Open Science Grid consortium for providing resources and support. This work was supported in part by the Offices of NP and HEP within the U.S. DOE Office of Science, the U.S. NSF, the Sloan Foundation, the DFG cluster of excellence "Origin and Structure of the Universe", CNRS/IN2P3, STFC and EPSRC of the United Kingdom, FAPESP CNPq of Brazil, Ministry of Ed. and Sci. of the Russian Federation, NNSFC, CAS, MoST, and MoE of China, GA and MSMT of the Czech Republic, FOM and NOW of the Netherlands, DAE, DST, and CSIR of India, Polish Ministry of Sci. and Higher Ed., Korea Research Foundation, Ministry of Sci., Ed. and Sports of the Rep. Of Croatia, Russian Ministry of Sci. and Tech, and RosAtom of Russia.

[1] C. S. Wu, E. Ambler, R. W. Hayward, D. D. Hoppes, and R. P. Hudson, Phys. Rev. 105, 1413 (1957).

[2] R. D. Peccei, Lect. Notes Phys. 741, 3 (2008).

[3] C. A. Baker et al., Phys. Rev. Lett. 97, 131801 (2006).

[4] D. Kharzeev, R. D. Pisarski, and M. H. G. Tytgat, Phys. Rev. Lett. 81, 512 (1998).

[5] D. Kharzeev, Phys. Lett. B 633, 260 (2006).

[6] D. E. Kharzeev, L. D. McLerran, and H. J. Warringa, Nucl. Phys. A 803, 227 (2008).

[7] K. Fukushima, D. E. Kharzeev, and H. J. Warringa, Phys. Rev. D 78, 074033 (2008).

[8] A. M. Poskanzer and S. A. Voloshin, Phys. Rev. C 58, 1671 (1998).

[9] S. A. Voloshin, Phys. Rev. C 70, 057901 (2004).

[10] B. I. Abelev et al., Phys. Rev. Lett. 101, 252301 (2008).

[11] B. I. Abelev et al., arXiv:0909.1717.

[12] K.H. Ackermann et al., Nucl. Instrum. Methods Phys. Res., Sect. A 499, 624 (2003).

[13] J. Adams et al., Phys. Rev. C 73, 034903 (2006). 
[14] I. Selyuzhenkov and S. Voloshin, Phys. Rev. C 77, 034904 (2008).

[15] N. Borghini, P. M. Dinh, and J. Y. Ollitrault, Phys. Rev. C 66, 014905 (2002).

[16] M. Gyulassy and X.-N. Wang, Comput. Phys. Commun. 83, 307 (1994); X. N. Wang and M. Gyulassy, Phys. Rev. D 44, 3501 (1991).
[17] J. Adams et al., Phys. Rev. Lett. 91, 172302 (2003).

[18] J. Adams et al., Phys. Rev. Lett. 95, 152301 (2005).

[19] L. Foa, Phys. Rep. 22, 1 (1975).

[20] B. Alver et al., Phys. Rev. C 75, 054913 (2007).

[21] M. Daugherity, J. Phys. G 35, 104090 (2008).

[22] R. L. Ray and R. S. Longacre, arXiv:nucl-ex/0008009.

[23] S. A. Bass et al., Prog. Part. Nucl. Phys. 41, 255 (1998). 